\title{
CHEMICALLY DECOUPLED NUCLEI IN DISK GALAXIES
}

\author{
SIL'CHENKO O.K. \\ Sternberg Astronomical institute, Moscow, Russia
}

In 1993 I have started an observational program to search for decoupled galactic nuclei with the Multi-Pupil Field Spectrograph (MPFS) of the $6 \mathrm{~m}$ telescope (Afanasiev et al. 1990); a list of 34 northern candidate galaxies with photometrically distinct red nuclei (Sil'chenko 1994) is used.

The investigation of a decoupled nucleus now includes observations with the MPFS in the green $(\lambda \lambda 4800-5400)$ and in the red $(\lambda \lambda 6200-6900)$ to construct maps of continuum and emission-line intensities, of ionized-gas and stellar velocities, as well as radial profiles of absorption-line equivalent widths. The primary reduction is made with the software developed in the Special Astrophysical Observatory (Vlasyuk 1993). The analysis of 2D velocity fields consists of looking for local velocity extremes inside a few arcseconds from the nuclei and of verifying a circular character of the rotation. The latter is made by examining an azimuthal dependence of observed central line-of-sight velocity gradients. If circular character of rotation is proved, we interpret an existence of circumnuclear velocity extremes as evidence for dynamical decoupling. As for chemically distinct nuclei, we detect them as abrupt drop of absorption-line strengths on the radial profiles. Now more than a half of all candidates are studied, a dozen of new certain chemically decoupled nuclei are found. Below are some examples.

Three isolated regular spiral galaxies, NGC 7331, 5533, and 4826, demonstrate a chemical distinctness of their unresolved nuclei in combination with circular planar corotation of gas and stars in the centers. NGC 2841 is also a Sb isolated regular galaxy, but its investigation with the $6 \mathrm{~m}$ and $1 \mathrm{~m}$ telescopes of SAO RAS has revealed particular properties of the central part (Sil'chenko et al. 1997). Besides the unresolved chemically decoupled nucleus presence, the ionized gas inside $R=4$ " rotates orthogonally to star rotation, and some signs of kinematically decoupled stellar component in the bulge are also present. After findings in NGC 2841, two lenticular polar-ring galaxies were studied, NGC 2685 and IC 1689. The chemically decoupled 
nuclei are detected in both of them; an age distinctness is also possible for the nucleus in IC 1689 (Sil'chenko 1998).

We can point out two common features which may be related to an origin of chemically decoupled nuclei.

Firstly, almost all the galaxies are known to have dynamically decoupled stellar and/or gaseous subsystems. NGC 4826 has two counterrotating gaseous disks (Braun et al. 1992, 1994; Rubin 1994; Rix et al. 1995). NGC 2841, 2685, and IC 1689 have polar gaseous disks of different sizes. NGC 7331 (Prada et al. 1996) and 2841 have "counterrotating" bulge components. In some cases multi-component structure is seen on photometric data: NGC 5533 and 7331 have two exponential disks, inner and outer.

Secondly, often an age difference between stellar populations of a chemically decoupled nucleus and of its outskirts can be detected: stars of the chemically decoupled nuclei are on the average younger.

This set of observational properties allows us to suggest what may result in chemical distinctness of nuclei. Multiple gaseous subsystems imply gas accretion events. Multiple stellar subsystems imply several discrete star formation epochs. Younger ages of stars in decoupled nuclei imply one of the latest star formation epochs to be localized in the nuclei. So we suppose that a galaxy had accreted once a large gaseous cloud. In a spiral galaxy with its own large-scale gaseous disk, this disk had to be strongly turbulized, and the own gas of the galaxy had to fall partly into the center having provoked a secondary nuclear star formation burst which produced new high-metallicity star generation looking now as a chemically decoupled nucleus. Accreted gas was initially beyond the global disk of the galaxy, so its drift to the center was not so effective due to smaller viscosity and dynamical friction. It is more probable that a final distribution of the accreted gas may be extended enough; it could be transformed into stars (NGC 2841 and 7331) or remain in gaseous form (NGC 4826).

The work was supported by the grant of the Russian Foundation for Basic Research No. 95-02-04480.

\section{References}

Afanasiev V.L., Dodonov S.N.,, Sil'chenko O.K., Vlasyuk V.V., 1990, Prepr. SAO \# 54. Braun R., Walterbos R.A.M., Kennicutt R.C., Jr., 1992, Nature, 360, 442

Braun R., Walterbos R., Kennicutt R.C., Jr., Tacconi L.J., 1994, ApJ, 420, 558

Prada F., Gutierrez C.M., Peletier R.F., McKeith C.D., 1996, ApJ, 463, L9

Rix H.-W. R., Kennicutt R.C., Jr., Braun R., Walterbos R.A M., 1995, ApJ, 438, 155

Rubin V.C., 1994, AJ, 107, 173

Sil'chenko O.K., 1994, AZh, 71, 706

Sil'chenko O.K., Vlasyuk V.V., Burenkov A.N., 1997, A\&A, 326, 941

Sil'chenko O.K., 1998, A\&A, accepted

Vlasyuk V.V., 1993, Astrofiz. issled. (Izv. SAO RAS), 36, 107 